\title{
Correction to: Generative image completion with image-to-image translation
}

\author{
Shuzhen $\mathrm{Xu}^{1}$ (D) $\cdot$ Qing $\mathrm{Zhu}^{1} \cdot$ Jin Wang ${ }^{1}$
}

Published online: 24 July 2020

(C) Springer-Verlag London Ltd., part of Springer Nature 2020

\section{Correction to: Neural Computing and Applications (2020) 32:7333-7345 \\ https://doi.org/10.1007/s00521-019-04253-2}

Unfortunately, the corresponding author of this paper was incorrectly published as Shuzhen $\mathrm{Xu}$ in the original publication. The correct corresponding author should be Jin Wang.

In the original publication, the author affiliation was incorrectly published. The correct affiliation is as follows:

Faculty of Information Technology, Beijing University of Technology, Beijing 100124, People's Republic of China.
The original version of the acknowledgement unfortunately contained a mistake. The correct version is as follows:

This work was supported by Natural Science Foundation of China (61906008), Scientific Research Project of Beijing Educational Committee (KM202010005013), Beijing Natural Science Foundation (4164079, 4152008), National Key Research and Development Plan of China (2017YFF0211801) and the Opening Project of Beijing Key Laboratory of Internet Culture and Digital Dissemination Research.

Publisher's Note Springer Nature remains neutral with regard to jurisdictional claims in published maps and institutional affiliations.

The original article can be found online at https:// doi.org/10.1007/s00521-019-04253-2.

\footnotetext{
Jin Wang

ijinwang@bjut.edu.cn

Shuzhen Xu

xhbcdjsh@emails.bjut.edu.cn

Qing Zhu

ccgszq@bjut.edu.cn

1 Faculty of Information Technology, Beijing University of Technology, Beijing 100124, People's Republic of China
} 